\title{
European Center for Nanotoxicology - Nanoscale Materials: A New Challenge for Toxicology
}

\author{
F. SinNer $^{1,2}{ }^{\text {, E. ROBLegG }}{ }^{3}$, E. FröHLICH ${ }^{4}$, A. ZIMmer ${ }^{3}$, A. FALK ${ }^{1}$ \\ ${ }^{1}$ BioNanoNet Forschungsgesellschaft $\mathrm{mbH}$, Graz, Austria \\ ${ }^{2}$ Health - Institute of Biomedicine and Health Sciences, Joanneum Research Graz, Austria \\ ${ }^{3}$ Institute of Pharmaceutical Sciences/ Pharmaceutical Technology, Karl-Franzens University Graz, Austria \\ ${ }^{4}$ Center for Medical Research, Medical University of Graz, Austria \\ E-mail: frank.sinner@bionanonet.at (F. Sinner)
}

Sci Pharm. 2010; 78: 580

doi:10.3797/scipharm.cespt.8.LNT06

Introduction: Nanotechnologies and Nanomedicine are promising fields of scientific research. Experts expect a huge economic and social impact from Nanotechnologies and Nanomedicine in the next decades. New properties associated with size smaller than $100 \mathrm{~nm}$ opens a new world of applications. At the same time, possible toxicological aspects associated with nanotechnologies are being discussed in the scientific community. Nanotoxicity, possibly resulting from altered chemical and physical behaviour of nanoparticles compared to bulk material, is a major concern nowadays. These phenomena are believed to be related to the extreme high specific surface area and altered electrochemical properties of nanoparticles. The investigation of nanomaterials in terms of their toxicological behaviour is an extremely multidisciplinary challenge. Toxicology of non-nano chemicals is a well established procedure. Unfortunately, Nanotoxicology is quite more complicated and standard protocols in Toxicology can not be used. Nanomaterials have to be investigated in detail for different parameters like size, size distribution, shape, charge, etc. Furthermore, interaction with different matrices like blood, interstitial fluid, buffers, etc. influences significantly nanomaterials like, agglomeration, size distribution, charge, etc. In consequence, nanotoxicology needs new standardised protocols which are able to deal with the extreme complexity of nanomaterials and their toxicological behaviour!

Focus: To build a national contact point for nanotoxicology, the BioNanoNet as a network company, has initiated the build up of the European Center of Nanotoxicology (www.euro-nanotox.at). This virtual center unites all Austrian Experts in the field of Nanotoxicology and helps to develop standardized methods. EURO-NanoTox initiates research projects together with its members and is links Austrian activities in the field of nanotoxicology to European activities.

Within this contribution the European Center for Nanotoxicology will be presented. The general strategy of a stepwise approach to investigate nanomaterials in respect of their potential toxic properties will be shown. Furthermore, some details for the investigation of skin penetration of quantum dots will be addressed. 